\title{
Análisis microscópico de clinker para optimizar el proceso de clinkerización en una fábrica cementera.
}

\begin{abstract}
(c) (i) () (2) BY NC SA

\section{Microscopic analysis of clinker to optimize the clinkerization process in a cement factory.}

Ana Gabriela Flores Huilcapi. ${ }^{1}$, Luis Santiago Carrera Almendáriz. ${ }^{2}$ \& Adrián Alejandro Rodríguez Pinos. ${ }^{3}$

Recibido: 10-11-2019 / Revisado: 05-12-2019 /Aceptado: 22-12-2019/ Publicado: 04-01-2020

\begin{abstract}
.
DOI: $\underline{\text { https://doi.org/10.33262/cienciadigital.v4i1.1090 }}$

The objective of this research was to optimize the clinkering process by means of microscopic analysis of clinker, to improve its quality, increasing C3S (alite) and diminishing $\mathrm{C} 2 \mathrm{~S}$ (belite) of its mineralogical composition, in cement factory. The clinker systematically tested for 12 weeks, was analyzed in the Quality Control Laboratory by microscopy. The interpretation of the texture, distribution and quantification of its mineral phases, let us to determine the stages of the clinkering process to be studied, as: Content of $\mathrm{CaCO} 3$ in the pre-homogenization of to the limestone, grinding and homogenization of raw, cooling temperature of the clinker and speed of the oven. The parameters of the processes were registered statistically by 6 weeks. This monitoring determines to adjust the parameters of quality as lime saturation factor on 94,20 and the grinding and homogenization of raw processes that keep on 20,44\% Retained M:200 and 2,11 std, respectively, which produce a clinker with $56,17 \% \mathrm{C} 3 \mathrm{~S}$ (alite) and $24,08 \% \mathrm{C} 2 \mathrm{~S}$ (belite), average of mineral phases. As a result of the adjustment to 99,94 lime saturation factor, 18,25 Retained M:200 and 1,83
\end{abstract}

1 Escuela Superior Politécnica de Chimborazo, Facultad de Mecánica, Riobamba, Ecuador, ana.flores@espoch.edu.ec

2 Escuela Superior Politécnica de Chimborazo, Facultad de Ciencias, Riobamba, Ecuador, luissantiago.carrera@espoch.edu.ec

3 Escuela Superior Politécnica de Chimborazo, Facultad de Ciencias, Riobamba, Ecuador, adrian.rodriguez@espoch.edu.ec 
std in the grinding and homogenization of raw processes, respectively, in the following 6 weeks, it increases in $8 \%$ of C3S and it diminishes in 10,13\% C2S in its mineral phases, improving the quality of the clinker, because it develops better mechanical resistances of the final product as it is the cement.

Keywords: Clinker, Microscopy, Alite, Belite

\section{Resumen.}

El objetivo de esta investigación fue optimizar el proceso de clinkerización mediante análisis microscópico de clínker, para mejorar su calidad, aumentando $\mathrm{C}_{3} \mathrm{~S}$ (alita) y disminuyendo $\mathrm{C}_{2} \mathrm{~S}$ (belita) de su composición mineralógica en una fábrica cementera. El clínker muestreado sistemáticamente durante 12 semanas fue analizado en el Laboratorio de Control de Calidad por microscopía. La interpretación de la textura, distribución y cuantificación de sus fases minerales, permitieron determinar las etapas del proceso de clinkerización a ser estudiadas como: Contenido de $\mathrm{CaCO}_{3}$ en la prehomogenización de la caliza, molturación y homogeneización de crudo, temperatura de enfriamiento del clínker y velocidad del horno. Los parámetros de los procesos mencionados son registrados y tratados estadísticamente por 6 semanas. Este monitoreo determina ajustar los parámetros de calidad como el factor de saturación de cal en promedio de 94,20 y de los procesos de molturación y homogeneización de crudo que mantienen 20,44\% Retenido M:200 y 2,11 std respectivamente, produciendo un clínker con $56,17 \% \mathrm{C}_{3} \mathrm{~S}$ (alita) y $24,08 \% \mathrm{C}_{2} \mathrm{~S}$ (belita) promedios de fases minerales. Como resultado del ajuste a 99,94 de factor de saturación de cal, 18,25 \% Retenido M:200 y 1,83 std en los procesos de molturación y homogeneización de crudo respectivamente en las siguientes 6 semanas, se aumenta en $8 \% \mathrm{de} \mathrm{C}_{3} \mathrm{~S}$ y se disminuye en $10,13 \% \mathrm{C}_{2} \mathrm{~S}$ en sus fases minerales, mejorando la calidad del clínker, pues desarrolla mayores resistencias mecánicas tempranas del producto final como es el cemento.

Palabras claves: Clinker, Microscopía, Alita, Belita.

\section{Introducción.}

La industria del cemento es una de las de mayor crecimiento a nivel mundial. En los últimos años las empresas han mejorado su línea de producción mediante la implementación de nuevos sistemas tecnológicos, con el propósito de disminuir sus costos de producción, especialmente los energéticos y obtener un producto de óptima calidad.

El clinker de cemento Portland se fabrica cociendo una mezcla de piedra caliza, cuarzo, arcilla y hierro a una temperatura de aproximadamente $1450{ }^{\circ} \mathrm{C}$ en un horno rotatorio de proporciones apropiadas y homogeneizada. La sinterización parcial de los componentes 
minerales da como resultado la formación de los nódulos de clínker, que después del enfriamiento se trituran con sulfato de calcio (aproximadamente 3\% a 5\% en peso) para producir un cemento Portland. Los cementos mezclados o compuestos pueden contener mayores cantidades de piedra caliza, escoria, cenizas volantes o puzolanas naturales según las especificaciones norteamericanas y europeas, ASTM C150, ASTM C595, ASTM C1157 y EN197. El concreto, el producto final utilizado en la construcción, contiene cemento (7\% a $15 \%$ en volumen), agua (14\% a $18 \%$ ), aire ( $4 \%$ a $8 \%$ en volumen) y agregado (59\% a $75 \%$ por volumen), y a menudo materiales cementosos suplementarios, como piedra caliza molida, cenizas volantes y escoria de alto horno (Hosmatka, KerKhoff, \& Panarese, 2008) (Stutzman, 2012).

El clínker de cemento se compone de cuatro fases primarias y una serie de fases menores del proceso de trituración y molienda, que da como resultado el cemento. El silicato tricálcico $\left(\mathrm{Ca}_{3} \mathrm{SiO}_{5}-\mathrm{C} 3 \mathrm{~S}\right)$ es la fase predominante en el clínker moderno, que contribuye aproximadamente del $40 \%$ al $75 \%$ del volumen (en masa), y es responsable del volumen de la resistencia a la edad temprana ( $<28$ d) del hormigón endurecido. Es comúnmente llamado alita, haciendo referencia a la forma impura que se encuentra en el clínker industrial. La alita es uno de los minerales principales del clinker, exhibe una sección transversal hexagonal, se presenta como cristales euédricos a anédricos y puede contener inclusiones de otras fases. El grabado químico facilita su identificación mediante microscopía de luz reflejada (Campbell, 2012).

Dentro de las técnicas de control de calidad del cemento portland está la microscopía del clínker. Un método poco costoso y fácilmente realizable. Los laboratorios de las fábricas de cemento no han aprovechado plenamente todas las posibilidades que les ofrece la observación microscópica de los clínkeres para cuantificar sus diferentes fases minerales.

Mediante la composición química determinada en el clínker, se establece matemáticamente el porcentaje de las fases mineralógicas presentes, pero este cálculo es teórico como bien lo dice Bogue, es un cálculo virtual (Tobón \& López, 2007) (Crumbie, Walenta, \& Füllmann, 2006). Con el análisis microscópico se determina y cuantifica real y físicamente la distribución, el tamaño y la cantidad de las fases minerales presentes en el clínker. La abundancia de fases, la distribución y la textura del clínker de cemento reflejan la combinación de dosificación, molienda y homogeneización de las materias primas, y el historial de cocción y enfriamiento del proceso de clínker. (Virella, 1982) La capacidad de visualizar, registrar y cuantificar los atributos de composición y textura de fase del clínker permitió desarrollar una mejor comprensión de su producción y la capacidad de identificar problemas en la preparación y cocción de las materias primas. Hoy en día, la mayoría de las microscopías utilizan secciones pulidas de clínker y luz reflejada, y los métodos cuantitativos incluyen el análisis de conteo de puntos, así como el procesamiento y análisis de imágenes. El desarrollo de materiales de referencia de clínker certificados ha facilitado el desarrollo de 
los primeros métodos de prueba estándar para su microscopía y la difracción de rayos X en polvo. (Zementindustrie, 2011).

El clinker está constituido por minerales como el silicato tricálcico $\left(\mathrm{C}_{3} \mathrm{~S}\right)$ comúnmente llamado Alita, el silicato bicálcico $\left(\mathrm{C}_{2} \mathrm{~S}\right)$, aluminato tricálcico $\left(\mathrm{C}_{3} \mathrm{~A}\right)$ y ferroaluminato tetracálcico $\left(\mathrm{C}_{4} \mathrm{AF}\right)$. El principal constituyente es el $\mathrm{C}_{3} \mathrm{~S}$ y se compone de $73 \%$ de cal y $26 \%$ de ácido silícico. Este mineral presenta formas prismáticas rectangulares o hexagonales bien conformadas, con dimensiones que varían de 15 - a $50 \mu \mathrm{m}$, su formación completa se da cuando la cocción se lleva a una temperatura suficientemente elevada y en un buen tiempo. $\mathrm{El} \mathrm{C}_{3} \mathrm{~S}$ contribuye al as resistencias iniciales. El segundo mineral de importancia en el clinker es el $\mathrm{C}_{2} \mathrm{~S}$ o silicato bicálcico, está constituido por un $65 \%$ de cal y un $34 \%$ de ácido silícico; presenta formas redondeadas, cristales anchos de entre 30 a $50 \mu \mathrm{m}$. El desarrollo de sus resistencias es lento, si bien a largo plazo es capaz de alcanzar resistencias tan grandes por lo menos como la alita. Las fases alumínicas y ferríticas microcristalinas son consideradas frecuentemente como mas intersticial o matriz,. Ambas se forman a partir de la fase líquida o fundida del clínker en el enfriamiento de éste. (Labahn \& Kohlhaas, 1983)

La interpretación de los atributos de composición y textura de los minerales del clínker permite comprender la efectividad de las diferentes etapas de fabricación del clinker como la molturación del crudo (molienda de arcilla y piedra caliza), homogeneización del crudo, calentamiento y temperatura de sinterización y enfriamiento del clinker.

La molturación del crudo se refiere al grado de finura que deben tener las materias primas previo al proceso de homogeneización. Para una molturación adecuada se requiere que el tamaño de partículas sea menor a $100 \mu \mathrm{m}$, aunque para Sílice se considera que debe ser menor a $45 \mu \mathrm{m}$. Una molturación deficiente se caracteriza por presentar: Nidos de $\mathrm{C}_{2} \mathrm{~S}$ (belita) con todos sus bordes bien definidos y formas regulares, indicando la presencia de cuarzo tosco > a $45 \mu \mathrm{m}$ en la alimentación. Los nidos de belita son perjudiciales para la molienda del clínker así también para la resistencia del Cemento. (Zea-Garcia, Santacruz, Aranda, \& De la Torre, 2019) La homogeneización del crudo adecuada presenta las fases mineralógicas bien distribuidas. Una homogeneización defectuosa se caracteriza por presentar nidos de algunos componentes mineralógicos como alita, belita, cal libre y periclasa. Los nidos son irregulares a redondeados y de diverso tamaño de 180 a $4000 \mu \mathrm{m}$; nidos de cal libre de 90 a $800 \mu \mathrm{m}$. Los nidos, al igual que en la molturación, son perjudiciales, porque gastan mucha energía en la molienda del clinker y también disminuyen la resistencia del Cemento (Taylor, 1982). El calentamiento dentro del horno se produce entre 1200 y $1300{ }^{\circ} \mathrm{C}$, antes de la zona de sinterización y puede ser interpretado observando el tamaño cristalino de la Belita, determinando un calentamiento adecuado por cristales de tamaño 20 a $40 \mu \mathrm{m}(60 \mu \mathrm{m})$. La temperatura de sinterización puede ser interpretada en base al estudio del tamaño cristalino de la Alita, así:15 - $20 \mu \mathrm{m}$ excelente, se considera una temperatura de sinterización muy buenas (1300 a $1450{ }^{\circ} \mathrm{C}$ ). Nidos de Belita bastante grandes y con formas irregulares indican 
un clínker sobrecosido. El tiempo de Sinterización insuficiente puede ser relacionado con falta de fundentes, o con una velocidad muy rápida del horno. (Maki, Ito, Tanioka, Ohno, \& Fukuda, 1993) El enfriamiento dentro del horno se interpreta básicamente observando la descomposición de los cristales de Alita, aunque también los cristales de Belita y Periclasa pueden servir como parámetros de ayuda. El enfriamiento inadecuado se relaciona con una velocidad del horno inadecuada o también con la formación de anillos.

La presente investigación en una fábrica cementera consistió en registrar las variables de calidad y de proceso de producción del clínker y su análisis microscópico textural y cuantitativo de las principales fases minerales presentes en el clínker como: alita $\left(\mathrm{C}_{3} \mathrm{~S}\right)$, belita $\left(\mathrm{C}_{2} \mathrm{~S}\right)$, aluminato tricálcico $\left(\mathrm{C}_{3} \mathrm{~A}\right)$ y ferroaluminato tetracálcico $\left(\mathrm{C}_{4} \mathrm{AF}\right)$ durante 12 semanas continuas. Estos resultados están íntimamente relacionados con la pre-homogeneización de la caliza, grado de homogeneización y molturación del crudo, temperatura de enfriamiento del clínker, temperatura de enfriamiento y velocidad del horno (velocidad de giro). Al determinar mediante análisis microscópico resultados insatisfactorios en la composición, textura, tamaño y/o distribución de las fases minerales presentes en el clínker en las primeras 6 semanas, se tomarán acciones para corregir los parámetros de las variables afectadas de calidad y de proceso antes mencionados durante las siguientes 6 semanas para lograr optimizar el proceso de clinkerización.

\section{Metodología.}

El trabajo experimental se realiza durante 12 semanas; cada día se obtiene una muestra representativa de $2 \mathrm{~kg}$ de clinker compuestas por muestras tomadas cada hora a la salida del horno para determinar parámetros de calidad como composición química de crudo determinada por \% de carbonato de calcio, módulo de saturación de cal, módulos de silicio y módulo de alúmina, contenido de alita y belita calculado y por análisis microscópico. La muestra representativa de clinker diaria es pulverizada y analizada en un equipo de fluorescencia de rayos $\mathrm{X}$ para cuantificar la cantidad de $\mathrm{CaO}, \mathrm{SiO}_{2}, \mathrm{Al}_{2} \mathrm{O}_{3}, \mathrm{Fe}_{2} \mathrm{O}_{3}$ y $\mathrm{MgO}$ principalmente para calcular los módulos de clinker.

El módulo de saturación de cal es un indicador usado en control de calidad del crudo para determinar la cantidad de cal ideal, de tal manera que se formen componentes hidráulicos en el mineral de clinker, sin que quede óxido de calcio libre. Una cantidad adecuada de cal en el clínker permite formar fases que requieren de cal y que tienen propiedades favorables, en particular en el desarrollo de resistencias en el producto final como es el cemento. El módulo de saturación de cal a menudo está entre 90 y 102. (Labahn \& Kohlhaas, 1983) y viene dado por la siguiente fórmula:

S.C. $=100(\mathrm{CaO}+0,75 \mathrm{MgO}) /\left(2,80 \mathrm{SiO}_{2}+1,18 \mathrm{Al}_{2} \mathrm{O}_{3}+0,65 \mathrm{Fe}_{2} \mathrm{O}_{3}\right)$

Ec. 1 
El módulo de sílice establece la variación de fase fundida que se desarrolla en el horno durante la clikerización, es así como módulos altos altera la facilidad de cocción y por ende la exigencia de una mayor temperatura y energía para su fundición. Sus valores suelen estar entre 1,8 y 3,2. (Labahn \& Kohlhaas, 1983).

$\mathrm{MS}=\mathrm{SiO}_{2} /\left(\mathrm{Al}_{2} \mathrm{O}_{3}+\mathrm{Fe}_{2} \mathrm{O}_{3}\right)$

Ec. 2

El módulo de alúmina es un parámetro que determina la cantidad de fase fundida que tendrá el clinker. La relación entre el óxido de aluminio y el óxido de hierro influye en las características de la fase líquida del clinker como su viscosidad, cantidad y composición. Su valor suele estar entre 0,7-2,5. El incremento en el módulo resulta en un clinker duro, que requiere mayor energía para su molienda. Valores bajos de módulo aceleran la fragua del cemento. (Chinchón \& Sanjuan, 2008)

$\mathrm{MA}=\mathrm{Al}_{2} \mathrm{O}_{3} / \mathrm{Fe}_{2} \mathrm{O}_{3}$

Ec. 3

Así también se registra parámetros de proceso como grado de homogeneización (razón desviación estándar de \% de carbonato cálcico de crudo molino vs crudo horno), grado de molturación de crudo (\% retenido M:200), temperatura de enfriamiento de clinker y velocidad de horno (RPM) tomados directamente del proceso de fabricación

Para cuantificar la cantidad de alita y belita se puede realizar un cálculo potencial a través de las ecuaciones de Bogue. (Perry, 1982) A partir de la composición química de un clinker, es posible estimar su composición mineralógica, o sea su contenido de silicato tricálcico (alita), silicato bicálcito (belita), aluminato tricálcico y ferroaluminatotetracálcico, más sin embargo maneja varios errores:

$\mathrm{C}_{3} \mathrm{~S}=4,71 \mathrm{CaO}-7,602 \mathrm{SiO}_{2}-6,719 \mathrm{Al}_{2} \mathrm{O}_{3}-1,430 \mathrm{Fe}_{2} \mathrm{O}_{3}$

Ec. 5

$\mathrm{C}_{2} \mathrm{~S}=8,602 \mathrm{SiO}_{2}+5,068 \mathrm{Al}_{2} \mathrm{O}_{3}+1,079 \mathrm{Fe}_{2} \mathrm{O}_{3}-3,07 \mathrm{CaO}$

Ec. 6

De tal manera que la técnica de investigación que se va a emplear para determinar la forma, tamaño, distribución y cuantificación de minerales de alita $\mathrm{C}_{3} \mathrm{~S}$ y belita $\mathrm{C}_{2} \mathrm{~S}$ es microscopía de luz reflejada. Para el análisis microscópico se toman de cada muestra representativa 10 nódulos de clinker regular y se los coloca en un molde previamente engrasado. Mediante un equipo de vacío se coloca 28,8 gramos de resina epoxi y 11,2 de endurecedor previamente pesados y mezclados en el molde. Las probetas una vez seca, es desmoldada y cortada con el equipo IsoMet Low Speed.

Para el pulido de la probeta se utilizan diferentes paños siendo los siguientes: 
ISSN: 2602-8085

Vol. 4, $\mathrm{N}^{\circ} 1 .$, p. 165-181, enero - marzo 2020

Tabla 1. Paños utilizados para pulir superficies segmentadas de Clinker

\begin{tabular}{|c|c|c|c|c|c|}
\hline $\begin{array}{l}\text { Superficie } \\
\text { Paño }\end{array}$ & Lubricante & Abrasivo & $\begin{array}{c}\text { Tiempo } \\
\text { min }\end{array}$ & Fuerza & $\begin{array}{c}\text { Velocidad } \\
\text { RPM }\end{array}$ \\
\hline CARBMET & Metadi fluid & $\begin{array}{c}\mathrm{SiC}-400 \\
\text { Grit }\end{array}$ & $\begin{array}{c}\text { Liso } \\
\text { completamente }\end{array}$ & 7 & $220-250$ \\
\hline TEXMET2000 & Metadi fluid & $\begin{array}{c}\text { Metadi paste } \\
6 \mu \mathrm{m}\end{array}$ & 5 & 6 & $120-150$ \\
\hline TEXMET2000 & Metadi fluid & $\begin{array}{l}\text { Metadi paste } \\
1 \mu \mathrm{m}\end{array}$ & 4,5 & 5 & $12-150$ \\
\hline $\begin{array}{c}\text { MICROCLO } \\
\text { TH }\end{array}$ & $\begin{array}{l}\text { Isoprophyl } \\
\text { alcohol }\end{array}$ & Micropolish & $2,5-3,5$ & 3 & $100-120$ \\
\hline
\end{tabular}

Fuente: Laboratorio de Control de Calidad fábrica cementera

Las secciones pulidas son preparadas con los siguientes métodos de ataque químico de tal manera que se obtenga el tono de color deseado para las diferentes fases mineralógicas. Para lo cual se los coloca en un papel filtro sobre un vidrio plano, y se lo impregna con el reactivo de ataque por un tiempo determinado y se seca perfectamente la muestra con una corriente de aire caliente de 5 a 8 minutos.

Tabla 2. Reactivos químicos de ataque de superficie de probetas de Clinker

\begin{tabular}{|c|c|}
\hline Reactivo & Tipo de ataque \\
\hline Aire & $\begin{array}{l}\text { Luego de } 48 \text { horas se produce un ataque a la cal libre dando un } \\
\text { color café - amarillento. }\end{array}$ \\
\hline Agua destilada & $\begin{array}{l}\text { Durante } 5 \mathrm{~s} \text { ataca a la cal libre logrando colores de } \\
\text { rojo a amarillo rojizo; por un tiempo de } 20 \text { s ataca al aluminato } \\
\text { tricácico. }\end{array}$ \\
\hline $\begin{array}{l}\text { Nital (HNO3 al } 1 \% \text { en } \\
\text { Alcohol Etílico) }\end{array}$ & $\begin{array}{l}\text { Durante } 10 \text { s ataca a los silicatos y en menor medida a los } \\
\text { aluminatos y ferritos. }\end{array}$ \\
\hline Etanol + Agua (1:1) & $\begin{array}{l}\text { Ataque durante } 2 \mathrm{~s} \text { y con una disolución al } 1 \% \text { de } \mathrm{HC} 1 \text { en etanol } \\
\text { durante } 2 \mathrm{~s} \text { más reaccionan los aluminatos y ferritos. }\end{array}$ \\
\hline Agua destilada & $\begin{array}{l}\text { Durante } 2 \text { s, más Nital por } 2 \mathrm{~s} \text { y } \mathrm{NH}_{4} \mathrm{Cl} \text { de } 30 \text { a } 45 \mathrm{~s} \text { se revelan } \\
\text { minerales como } \mathrm{C}_{3} \mathrm{~S}, \mathrm{C}_{2} \mathrm{~S} \text { y } \mathrm{C}_{3} \mathrm{~A} \text {. }\end{array}$ \\
\hline
\end{tabular}

Fuente:(Fernández, 2009)(Dorn, 1978) 


\section{Resultados.}

La saturación de cal durante las primeras 6 semanas mantienen un promedio de 94,20 considerado en el límite bajo para la formación de las fases ricas en cal responsables de las resistencias iniciales del cemento por lo que se ajusta este parámetro en la química del crudo y durante las siguientes 6 semanas se sube la saturación de cal promedio a 99,94 considerado de alta calidad. El módulo de alúmina decrece de 1,59 a 1,53 entre los dos períodos proporcionando una mayor cantidad de fase fundida que resulta en la formación de cristales de tamaño y bordes regulares.

Tabla 3. Promedio semanal de variables de calidad de composición química de clinker

\begin{tabular}{ccccc}
\hline Semana & $\begin{array}{c}\% \\
\text { Carbonato } \\
\text { de Calcio }\end{array}$ & $\begin{array}{c}\text { Saturación } \\
\text { de cal }\end{array}$ & $\begin{array}{c}\text { Módulo de } \\
\text { Silicio }\end{array}$ & $\begin{array}{c}\text { Módulo de } \\
\text { Alúmina }\end{array}$ \\
\hline 1 & 81,77 & 93,23 & 2,9 & 1,60 \\
2 & 81,76 & 92,31 & 2,74 & 1,63 \\
3 & 82,06 & 91,87 & 2,78 & 1,68 \\
4 & 82,36 & 92,73 & 2,82 & 1,57 \\
5 & 82,42 & 95,61 & 2,57 & 1,55 \\
6 & 81,85 & 99,47 & 2,65 & 1,49 \\
7 & 80,62 & 103,44 & 2,78 & 1,48 \\
8 & 81,78 & 103,86 & 2,58 & 1,50 \\
9 & 79,30 & 100,86 & 2,49 & 1,56 \\
10 & 80,01 & 97,33 & 2,73 & 1,58 \\
11 & 80,16 & 95,81 & 3,02 & 1,57 \\
12 & 82,68 & 98,31 & 2,89 & 1,50 \\
std & 1,45 & 4,527 & 0,19 & 0,08 \\
\hline
\end{tabular}

Fuente: Elaboración propia

En las primeras 6 semanas por microscopía se observa la presencia de nidos de belita con bordes bien definidos en los nódulos de clinker como se observa en la Fig. 4 , este tipo de alteración mineralógica está relacionada con procesos poco efectivos en cuanto a homogeneización y molturación de crudo, según la Tabla se tiene un promedio de 2,11 std y 20,44\% de Retenido M:200 respectivamente, estos datos demuestran que están sobre el rango óptimo de proceso de manera que se ajusta estos parámetros en las siguientes 6 semanas bajando el porcentaje de Retenido M:200 a un promedio de 18,25\% y una homogeneización de 1,83 std en el crudo, inclusive se puede observar que en el promedio de la semana 7 se baja bruscamente el retenido M:200 a 9,91 debido a que no se logra estandarizar las variables del de operación del molino de crudo, situación que se controla en las siguientes semanas obteniendo un retenido en M:200 estable. 
La Temperatura de enfriamiento en las primeras semanas está en un promedio de 80,10 C, valores considerados dentro de lo esperado y contrastado con el análisis microscópico de clinker se observa que el enfriamiento es rápido ya que se observa alitas con forma romboédricas, bordes bien definidos y rectos, mientras que la belita mantiene una forma redondeada y de tamaño pequeño, y no se observa descomposición de alitas en belitas. Esta variable de proceso en las siguientes 6 semanas se baja a un promedio de 69,91 C de manera que se mejora aún más la calidad de los minerales.

Tabla 4. Promedio semanal de minerales de C3S y C2S calculado y analizado por microscopía de clinker

\begin{tabular}{ccccccc}
\hline Semana & $\begin{array}{c}\mathrm{C}_{3} \mathrm{~S} \\
\text { calculado } \\
\text { Bogue } \\
(\%)\end{array}$ & $\begin{array}{c}\mathrm{C}_{3} \mathrm{~S} \\
\text { cuantificado } \\
\text { microscopía } \\
(\%)\end{array}$ & $\begin{array}{c}\text { Tamaño } \\
\text { promedio } \\
\mathrm{C}_{3} \mathrm{~S} \\
(\mu \mathrm{m})\end{array}$ & $\begin{array}{c}\mathrm{C}_{2} \mathrm{~S} \\
\text { calculado } \\
\text { Bogue } \\
(\%)\end{array}$ & $\begin{array}{c}\mathrm{C}_{2} \mathrm{~S} \\
\text { cuantificado } \\
\text { microscopía } \\
(\%)\end{array}$ & $\begin{array}{c}\text { Tamaño } \\
\text { promedio } \\
\mathrm{C}_{3} \mathrm{~S} \\
(\mu \mathrm{m})\end{array}$ \\
\hline 1 & 50,47 & 53,2 & 40,34 & 27,69 & 28,68 & 36,1 \\
2 & 53,13 & 56,21 & 42,83 & 24,47 & 23,32 & 45,33 \\
3 & 54,06 & 57,75 & 43,51 & 23,67 & 21,85 & 37,5 \\
4 & 52,54 & 58,32 & 47,40 & 25,43 & 20,97 & 28,41 \\
5 & 55,6 & 58,32 & 35,21 & 22,14 & 20,97 & 29,02 \\
6 & 60,87 & 53,2 & 31,4 & 17 & 28,68 & 23,63 \\
7 & 67,01 & 70,35 & 26,70 & 9,77 & 8,57 & 35,21 \\
8 & 62,19 & 65,28 & 28,50 & 11,34 & 10,13 & 38,53 \\
9 & 63,31 & 66,15 & 31,26 & 12,06 & 11,89 & 39,38 \\
10 & 61,39 & 63,54 & 28,54 & 15,43 & 12,59 & 48,37 \\
11 & 59,74 & 58,35 & 36,84 & 17,63 & 16,89 & 38,22 \\
12 & 59,87 & 61,34 & 34,61 & 17,49 & 16,23 & 37,97 \\
std & 5,147 & 1,38 & 6,72 & 5,18 & 1,53 & 6,91 \\
\hline
\end{tabular}

Fuente: Elaboración propia

La cuantificación de C3S - Alita por el método calculado (bogue) arroja como datos un $2 \%$ menos que lo determinado por microscopía, mientras que la cuantificación de $\mathrm{C}_{2} \mathrm{~S}$ (belita) por el método calculado (bogué) muestra un $2 \%$ más que lo cuantificado por microscopía. El $\mathrm{C}_{3} \mathrm{~S}$ y $\mathrm{C}_{2} \mathrm{~S}$ promedio cuantificado por microscopía durante las 6 primeras semanas corresponde al 56, 17 \% y 24,08\% Al mejorar los parámetros de calidad de composición de crudo como el Factor de Saturación de Cal, Módulos de silicio y Módulos de alúmina y los parámetros de proceso como molturación de crudo, homogeneización de crudo y temperatura de enfriamiento de clinker se logra en las siguientes 6 semanas subir el C3S promedio a $64,17 \%$ y bajar el C2S promedio a $13,95 \%$, pues mientras mayor porcentaje se logre de C3S mejores resistencias iniciales se lograrán en el producto final como es el cemento. Así las resistencias a la compresión iniciales de cemento promedio de las 6 primeras 
semanas están en 13,17 MPa, mientras que en las 6 semanas siguientes con el aumento de C3S se obtiene unas resistencias a la compresión iniciales de cemento promedio de 15,10 MPa es decir se logra un aumento de $12,8 \%$ con la mejora de las variables de calidad y de proceso.

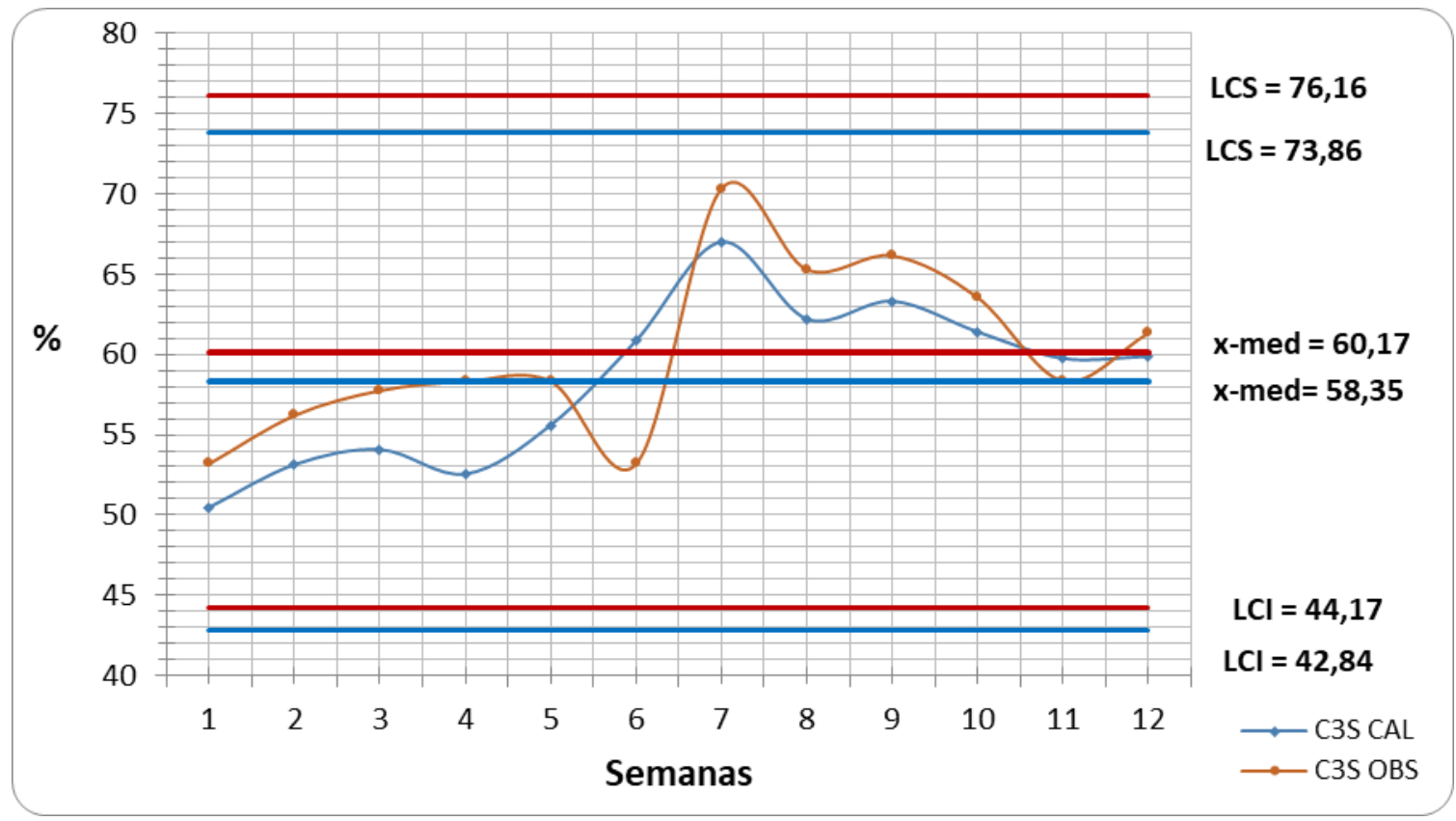

Figura 1. Carta de Control X-med de $\mathrm{C}_{3} \mathrm{~S}$ calculado y observado

Fuente: Elaboración propia

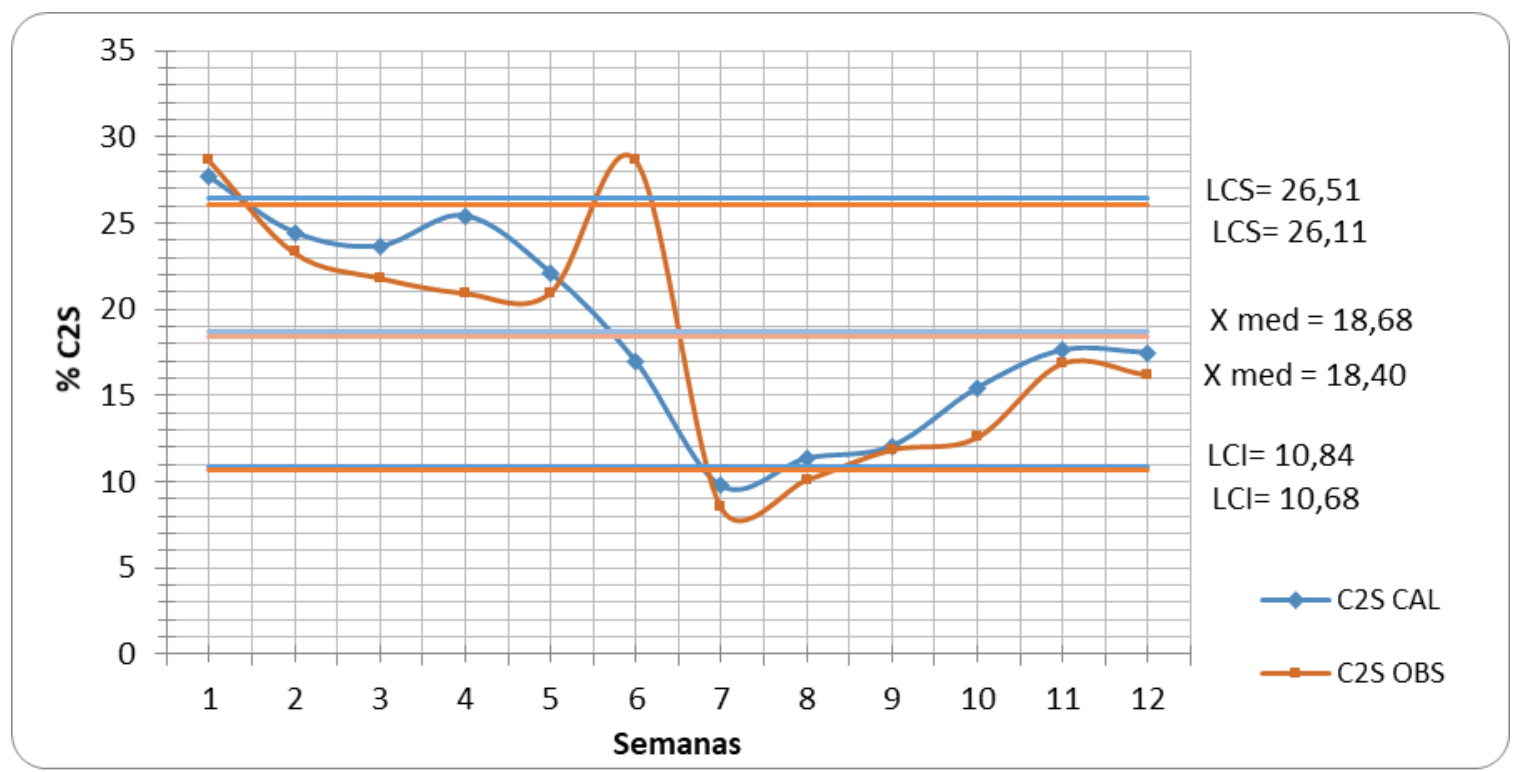

Figura 2. Carta de Control X-med $\mathrm{C}_{2} \mathrm{~S}$ observado y calculado clínker horno

Fuente: Elaboración propia 
Según la Figura 1 y la Figura 2 se puede apreciar que a partir de la séptima semana se mejora la composición de C3S y se disminuye la composición de C2S en el clinker. Además, se observa una diferencia significativa de porcentaje medido entre el método calculado (Bogué) y microscopía óptica.

Tabla 5. Promedio semanal de variables de proceso de clinker

\begin{tabular}{ccccc}
\hline Semana & $\begin{array}{c}\text { Grado homogeneización } \\
\text { Desviación estándar Crudo } \\
\text { molino/Crudo horno }\end{array}$ & $\begin{array}{c}\text { Grado molturación } \\
\text { de crudo } \\
\text { \% Retenido M:200 }\end{array}$ & $\begin{array}{c}\text { Temperatura } \\
\text { enfriamiento } \\
\text { clinker }\end{array}$ & $\begin{array}{c}\text { Velocidad } \\
\text { de horno } \\
\text { RPM }\end{array}$ \\
\hline 2 & 2,03 & 20,03 & 81,10 & 1,38 \\
3 & 2,27 & 19,94 & 77,89 & 1,41 \\
4 & 2,39 & 21,50 & 84,17 & 1,65 \\
5 & 1,71 & 20,67 & 92,16 & 1,46 \\
6 & 2,13 & 20,72 & 67,38 & 1,38 \\
7 & 2,17 & 19,79 & 77,9 & 1,58 \\
8 & 1,61 & 9,91 & 72,46 & 1,5 \\
9 & 2,24 & 21,04 & 72,54 & 1,29 \\
10 & 2,16 & 19,91 & 60,03 & 1,33 \\
11 & 1,63 & 20,13 & 59,38 & 1,70 \\
12 & 1,33 & 18,46 & 80,62 & 1,70 \\
std & 2,01 & 20,03 & 73,20 & 1,28 \\
\hline
\end{tabular}

Fuente: Elaboración propia

Tabla 6. Promedio semanal de resistencias a la compresión de cemento Resistencia a la compresión

\begin{tabular}{cccc}
\hline Semana & 3 días & 7 días & 28 días \\
\hline 1 & 10,50 & 17,38 & 29,78 \\
2 & 15,06 & 20,87 & 32,65 \\
3 & 13,27 & 20,01 & 29,96 \\
4 & 11,04 & 17,83 & 26,40 \\
5 & 12,32 & 19,15 & 28,14 \\
6 & 16,84 & 24,24 & 33,09 \\
7 & 15,90 & 21,19 & 30,86 \\
8 & 16,80 & 21,70 & 30,20 \\
9 & 16,64 & 22,46 & 30,51 \\
10 & 13,37 & 19,11 & 26,21 \\
11 & 13,68 & 20,06 & 28,99 \\
12 & 14,23 & 20,07 & 26,69 \\
std & 2,66 & 2,90 & 3,18 \\
\hline
\end{tabular}

Fuente: Elaboración propia 
El grado de molturación de crudo definido por el retenido en malla 200 según la Tabla 5, en la semana 7 disminuye en 9,91\%, debido a que se eleva la velocidad en el separador del molino de crudo, sin embargo, el crudo con una finura tan baja empieza a generar problemas de atascamiento en la torre de ciclones del precalentamiento del crudo, por lo que se decide mantener en $19 \%$ de retenido en M:200, parámetro que se normaliza en 2 semanas.

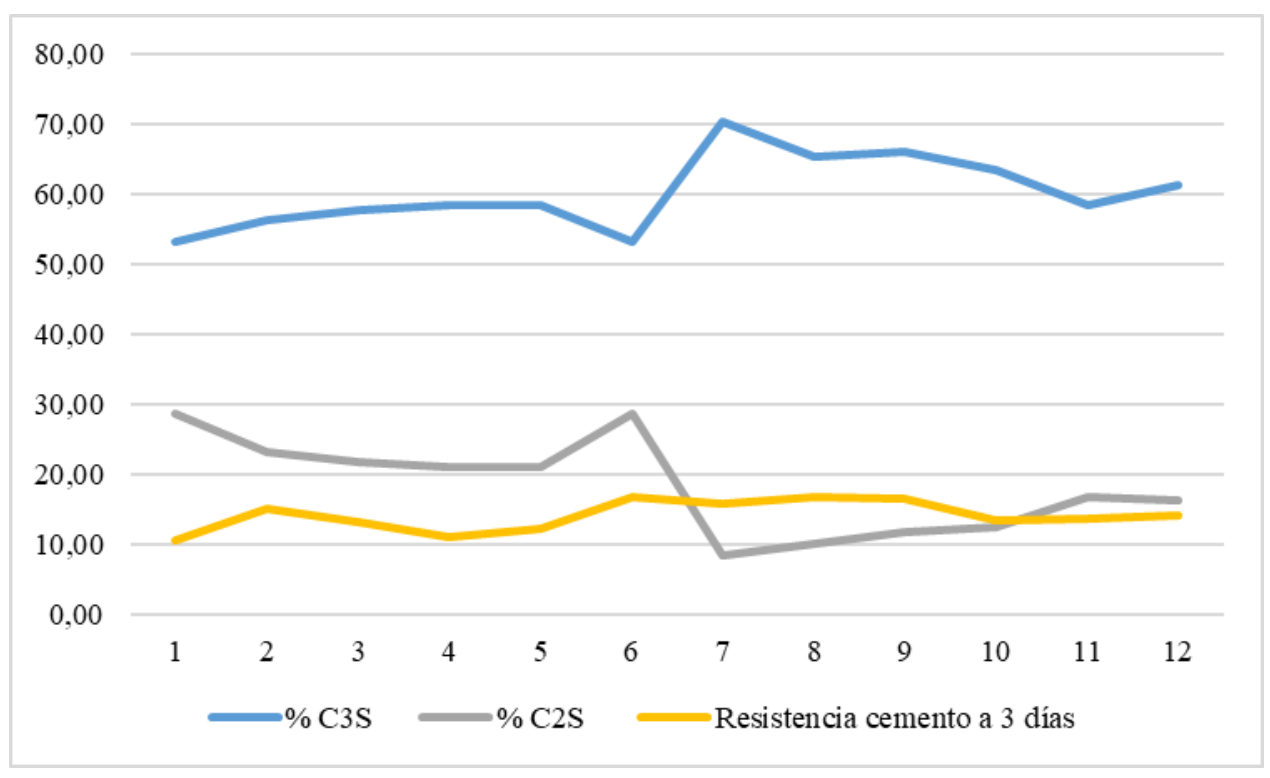

Figura 3. $\mathrm{C}_{3} \mathrm{~S}, \mathrm{C}_{2} \mathrm{~S}$ y resistencias iniciales a 3 días semanales promedio de clinker analizado por microscopía.

Fuente: Elaboración propia

Como se aprecia en la Figura 3 existe una relación estrecha entre el porcentaje de $C_{3} S$ cuantificado y las resistencias tempranas a 3 y 7 días en el producto final como es el cemento. Como se observa en la Figura 3 el incremento de $\mathrm{C}_{3} \mathrm{~S}$ a partir de las semanas 7, 8 y 9 de $67,01 \%, 62,19 \%$ y $63,31 \%$ respectivamente, provoca un aumento de las resistencias tempranas del cemento puzolánico IP a 3 días de 15,90, 16,80 y 16,64 Mpa respectivamente superando lo establecido por la Norma INEN 490:2 para Cementos Hidráulicos compuesto de $13 \mathrm{MPa}$. (INEN, 2002). 


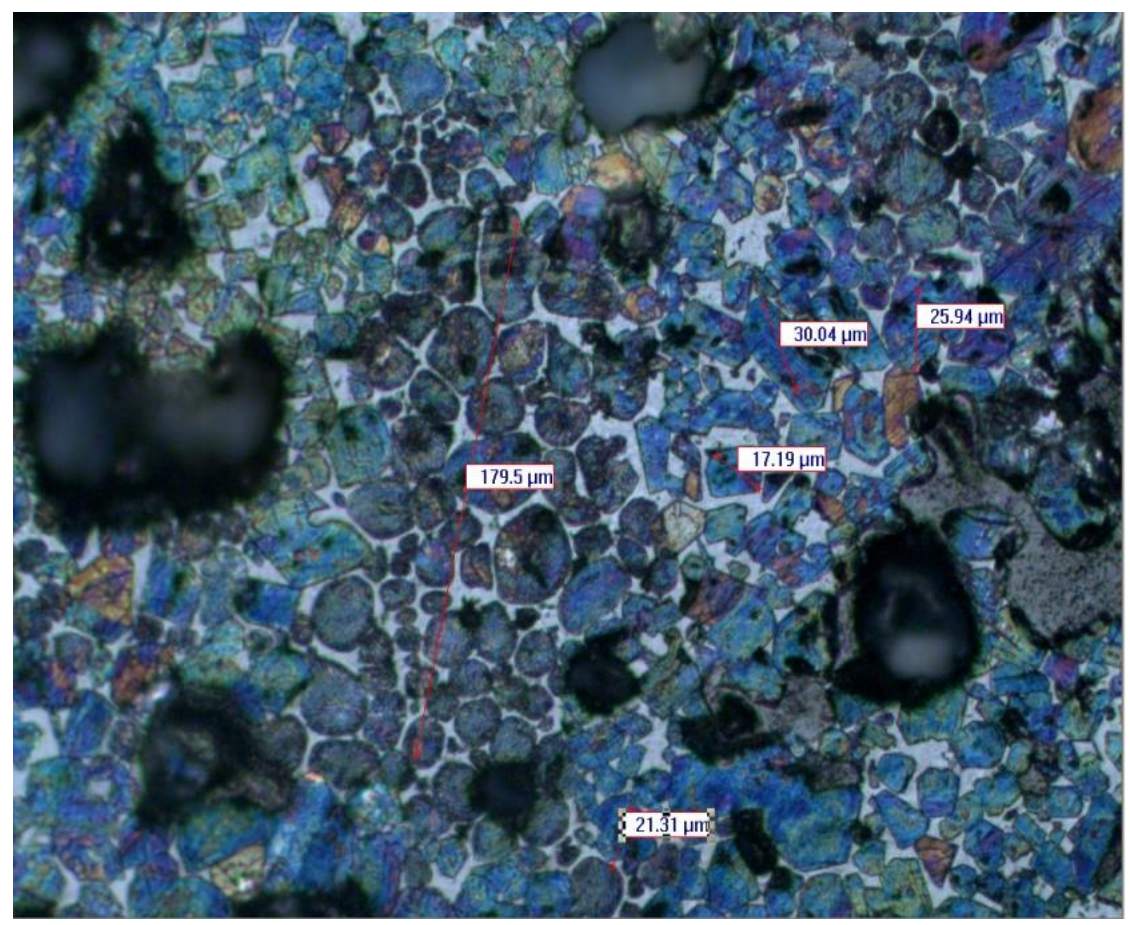

Figura 4. Análisis microscópico de clinker de semana 3 resolución $10 \mathrm{X}$ Fuente: Software Omniment de microscopía óptica

Se observa en la Figura 4 la presencia de nidos de $\mathrm{C}_{2} \mathrm{~S}$ (belita) de aproximadamente 179,5 $\mu \mathrm{m}$ y $\mathrm{C}_{3} \mathrm{~S}$ de $40,34 \mu \mathrm{m}$ promedio.

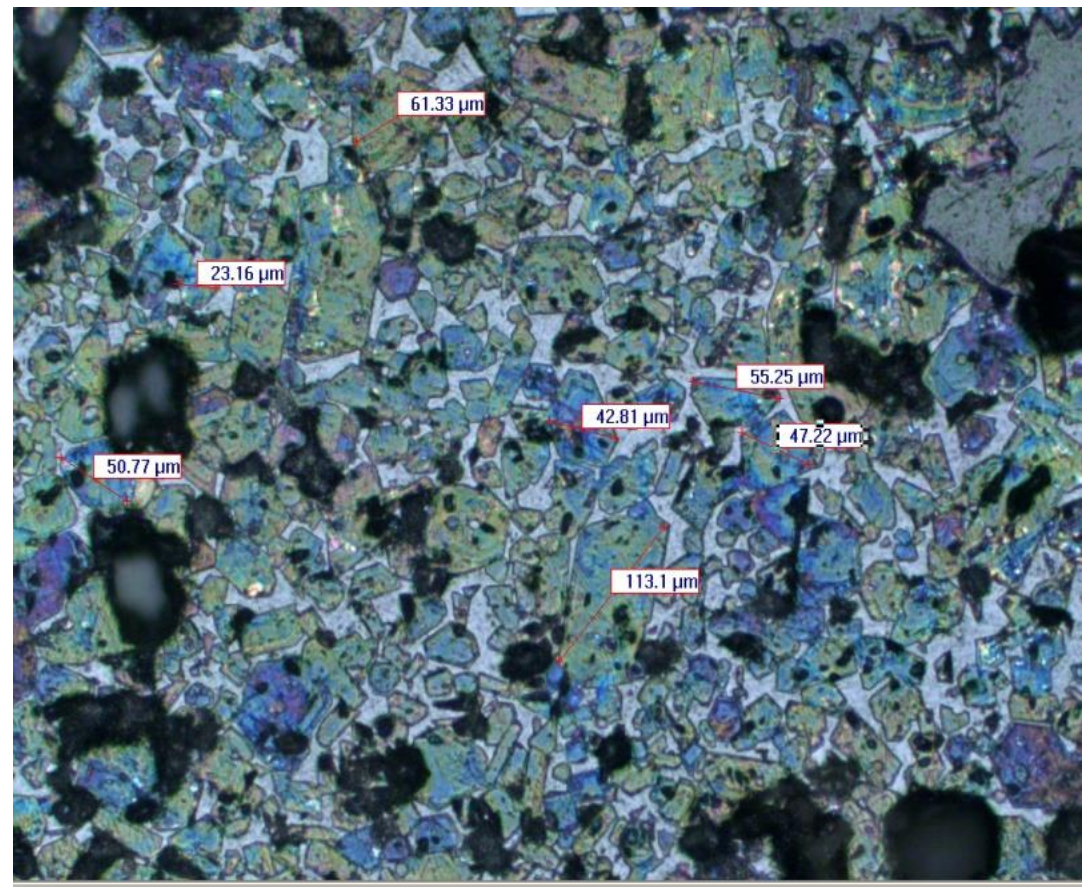

Figura 5. Análisis microscópico de clinker de semana 7 resolución $10 \mathrm{X}$ Fuente: Software Omniment de microscopía óptica 


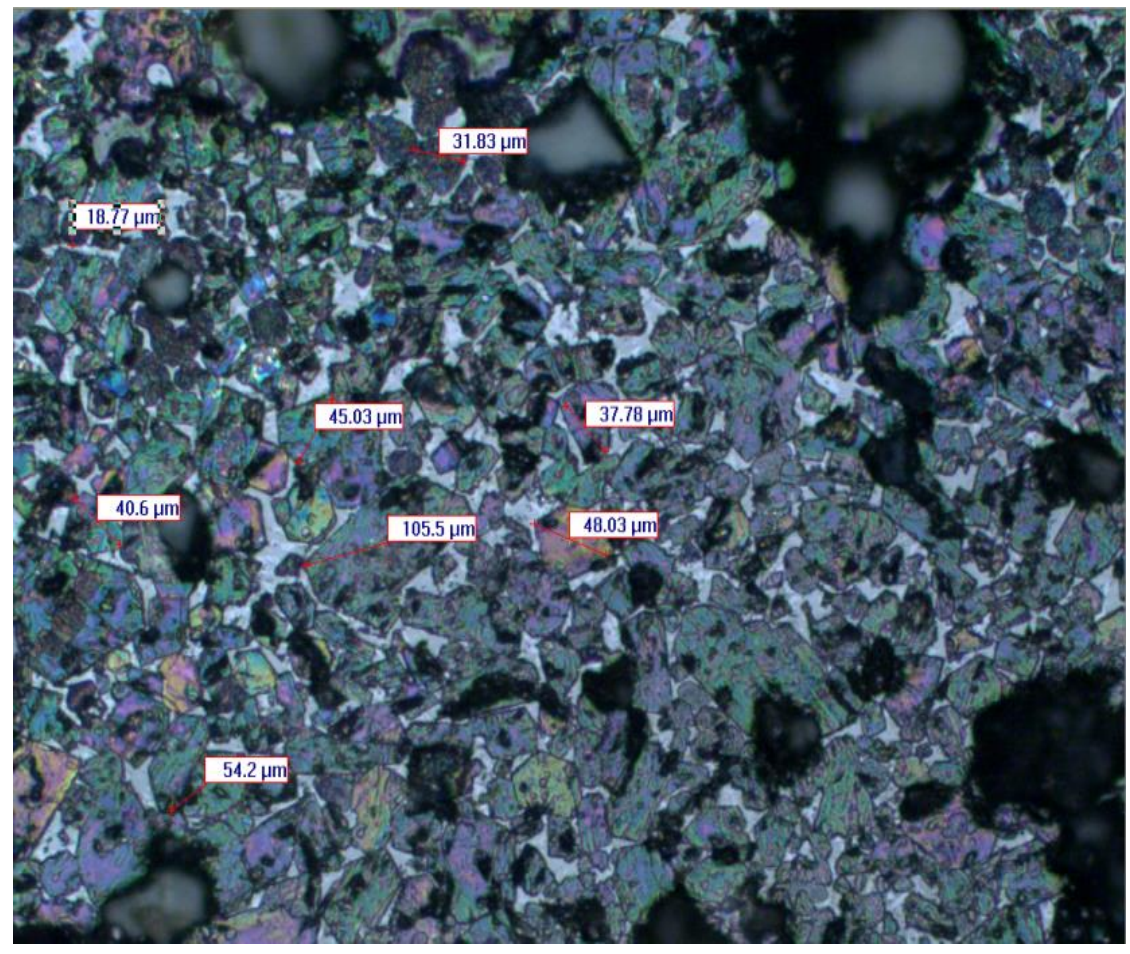

Figura 6. Análisis microscópico de clinker de semana 12 resolución $10 \mathrm{X}$ Fuente: Software Omniment de microscopía óptica

Una vez que se ha subido el factor de saturación de cal y se ha mejorado la finura del crudo y su homogeneización en las Figura 5 y 6 se observa mayor distribución de $\mathrm{C}_{3} \mathrm{~S}$, un tamaño promedio de $26,70 \mu \mathrm{m}$ y $34,61 \mu \mathrm{m}$ respectivamente, y no se aprecia la presencia de nidos de $\mathrm{C}_{2} \mathrm{~S}$ (belita).

\section{Conclusiones.}

- El factor de saturación de cal sobre 97 es adecuado para la formación de las fases minerales $\mathrm{C}_{3} \mathrm{~S}$ (alita) y $\mathrm{C}_{2} \mathrm{~S}$ (belita) del clínker, con módulos de silicio bajo los 2,8 y módulo de Alúmina alrededor de 1,5 obteniéndose una distribución en composición, forma y tamaño ideal de $\mathrm{C}_{3} \mathrm{~S}$ y $\mathrm{C}_{2} \mathrm{~S}$. El desarrollo bajo de las resistencias mecánicas tempranas del cemento se debe a un bajo contenido de $\mathrm{C}_{3} \mathrm{~S}$ (alita) y un alto porcentaje de $\mathrm{C}_{2} \mathrm{~S}$ (belita) según los resultados mostrados.

- La deficiencia en el grado de molturación y homogeneización se evidenció al tener microscopías de clinker que muestran la presencia de nidos de belita y cal libre irregulares que resultan perjudiciales porque gastan mucha energía en su molienda posterior y también disminuyen la resistencia del cemento.

- Las variables de proceso como temperatura del clínker en la etapa de enfriamiento y la velocidad del horno están controladas y sus valores son adecuados, ya que se evidencia un bajo porcentaje de silicato bicálcico (belita) secundaria y una matriz bien cristalizada. 
- Se cuantifica por microscopía óptica a través del software Omniment que el clínker de la fábrica cementera posee un $60,16 \%$ de $\mathrm{C}_{3} \mathrm{~S}$ y $18,39 \%$ de $\mathrm{C}_{2} \mathrm{~S}$ promedio, concluyéndose que el $\mathrm{C}_{3} \mathrm{~S}$ observado microscópicamente es mayor en un $2 \%$ que el calculado, mientras que $\mathrm{C}_{2} \mathrm{~S}$ observado y calculado tienen resultados aproximadamente iguales. El ajuste realizado en las variables de calidad como el factor de saturación de cal y variables de procesos de molturación y homogeneización de crudo permitieron que se suba la proporción de $\mathrm{C}_{3} \mathrm{~S}$ y se disminuya la cantidad de $\mathrm{C}_{2} \mathrm{~S}$ de sus fases minerales, mejorando la calidad del clínker.

\section{Referencias bibliográficas.}

Campbell, D. (2012). Microscopical Examination and Interpretation of Portland Cement and Clinker (2da ed.). Skokie/IL.

Chinchón, S., \& Sanjuan, M. (2008). El cemento de aluminato de calcio y sus prefabricados.

Crumbie, A., Walenta, G., \& Füllmann, T. (2006). Where is the iron? Clinker microanalysis with XRD Rietveld, optical microscopy/point counting, Bogue and SEM-EDS techniques. Cement and Concrete Research, 36(8), 1542-1547

Dorn, J. D. (1978). Microscopic methods for burnability improvement. Cement and Concrete Research, 8(5), 635-645.

Fernández, J. (2009). Microscopía del clínker de cemento portland. México: Patronato Juan De La Cierva De Investigación Científica y Técnica.

Hosmatka, H., KerKhoff, B., \& Panarese, W. (2008). Design and Control Design and Control of concrete mixtures (4ta ed.; P. C. Association, ed.). Illinois, USA.

Labahn, H., \& Kohlhaas, L. (1983). Prontuario del Cemento (5ta ed.). Barcelona, España: TécnicosAasociados.

INEN. Norma Técnica Ecuatoriana. Cementos Hidraúlicos Compuestos. Requisitos. , (2002

Maki, I., Ito, S., Tanioka, T., Ohno, Y., \& Fukuda, K. (1993). Clinker grindability and textures of alite and belite. Cement and Concrete Research, 23(5), 1078-1084.

Perry, R. (1982). Manual del Ingeniero Químico (2da ed.). México: Mc Graw Hill.

Stutzman, P. E. (2012). Microscopy of clinker and hydraulic cements. Reviews in Mineralogy and Geochemistry, 74(Bogue 1955), 101-146. Taylor, H. (1982). Determination of the quantitative phase composition of alite and belite in portland cement clinker by microphotometry. Cement and Concrete Research, 12(c), 511-516. 
Tobón, J., \& López, F. (2007). Adaptation of Bogue'S Equations To Mineralogical Estimate of the Clinker for a Colombian Cement Factory. Dyna, 74(153), 53-60.

Virella, A. (1982). Observación al microscopio del clinker de Cemento Portland. Madrid, España: Mc Graw Hill.

Zea-Garcia, J. D., Santacruz, I., Aranda, M. A. G., \& De la Torre, A. G. (2019). Alite-beliteye'elimite cements: Effect of dopants on the clinker phase composition and properties. Cement and Concrete Research, 115(October 2018), 192-202.

Zementindustrie, F. Der. (2011). Evaluation of Portland Cement Clinker With Optical Microscopy - Case Studies II. Building, (1), 1-7. 


\section{PARA CITAR EL ARTÍCULO INDEXADO.}

Flores Huilcapi, A. G., Carrera Almendáriz, L. S., \& Rodríguez Pinos, A. A. (2020). Análisis microscópico de clinker para optimizar el proceso de clinkerización en una fábrica cementera. Ciencia Digital, 4(1), 165-181. https://doi.org/10.33262/cienciadigital.v4i1.1090

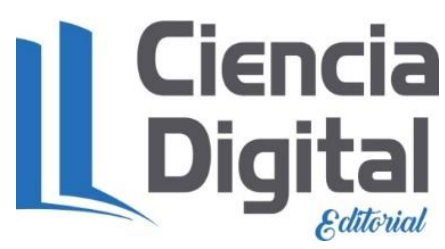

El artículo que se publica es de exclusiva responsabilidad de los autores y no necesariamente reflejan el pensamiento de la Revista Ciencia Digital.

El artículo queda en propiedad de la revista y, por tanto, su publicación parcial y/o total en otro medio tiene que ser autorizado por el director de la Revista Ciencia Digital.
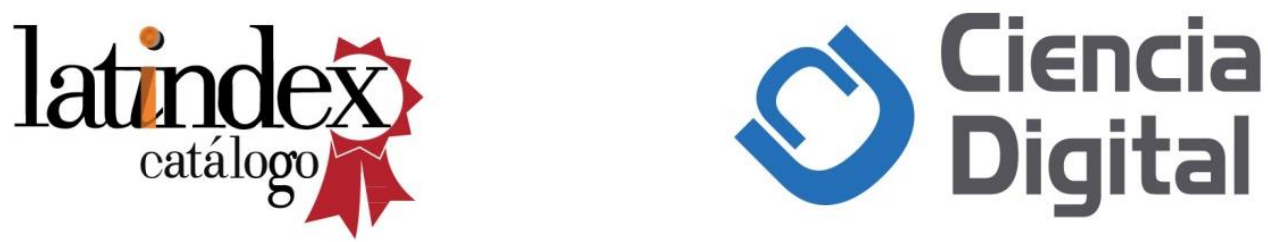